\title{
Perturbation Method in a Problem of Waveguide Theory
}

\section{David Fox and Wilhelm Magnus}

\author{
Contribution from New York University, Courant Institute of Mathematical Sciences, New York, N.Y.
}

(Received July 12, 1962; revised September 17, 1962)

\begin{abstract}
The reflection coefficient for the basic mode in a widening, straight, two-dimensional waveguide is computed for small wave numbers by using the perturbation method with the electrostatic case as the unperturbed case. The probiem is treated as a perturbed infinite system of inhomogeneous linear equations, and it is shown that the matrix of the unperturbed system (which corresponds to the electrostatic case) can be inverted explicitly by using conformal mappings and physically unrealistic modes. Questions of convergence are discussed, and other examples for application of the method are indicated.
\end{abstract}

\section{The Physical Problem}

\subsection{Structure}

The problem which we are considering is a twodimensional one arising from a three-dimensional waveguide structure in which the perfectly conducting surfaces extend from $-\infty$ to $\infty$ in the direction of the z-axis in a Cartesian coordinate system. The intersection of these conducting surfaces with the $x, y$-plane is given by the six lines:

$$
\begin{aligned}
& x \geq 0, y=q \pi \quad(0<q<1) \\
& x \geq 0, y=-q \pi \\
& x=0, q \pi \leq y \leq \pi \\
& x=0,-q \pi \geq y \geq-\pi \\
& x \leq 0, y=\pi \\
& x \leq 0, y=-\pi .
\end{aligned}
$$

The segment of the $y$-axis between $-q \pi$ and $q \pi$, which we shall refer to as the aperture, separates the waveguide structure into two simple regions, I and II.

\subsection{Conditions on the Electric Field}

Let the electric field $\vec{E}=\left(E_{x}, E_{y}, E_{z}\right)$, and assume that the time dependency is given by $e^{i \omega t}$ where

$\omega=k c, \quad k=$ wave number, $\quad c=$ velocity of light.

1 This research was supported by the Electronics Research Directorate of the Air Force Cambridge Research Laboratories, Office of Aerospace Research (USAF), Bedford, Mass., under Contract No. AF 19(604)5238.
Then we have the conditions

(1) $E_{x}, E_{y}$, and $E_{z}$ satisfy $\Delta u+k^{2} u=0$;

(2) $\frac{\partial E_{x}}{\partial x}+\frac{\partial E_{y}}{\partial y}+\frac{\partial E_{z}}{\partial z}=0$;

(3) the tangential component of $\vec{E}$ vanishes at the boundaries.

We shall also make the following assumptions:

(4) $E_{y}$ is an even function of $y$

(5) $E_{z} \equiv 0$.

The behavior of $\vec{E}$ at infinity must satisfy

(6) $\lim \left|E_{y}-\tau E_{o} e^{i k x}\right|=0, \quad E_{x} \rightarrow 0$

(7) $\lim \left|E_{y}-E_{o} e^{i k x}-\rho E_{o} e^{-i k x}\right|=0, \quad E_{x} \rightarrow 0$

(8) $\iint\left(E_{x}^{2}+E_{y}^{2}\right) d x d y$ is bounded over every finite region.

We shall introduce the following detinitions:

$\tau$ (condition (6)) is called the transmission coefficient.

$\rho$ (condition (7)) is called the reflection coefficient.

$E_{o} e^{i k x}$ is called the incident wave.

$E_{y}-E_{o} e^{i k x}$ is called the diffracted wave. 
We shall now show that the field is completely determined by $E_{y}$ alone. Conditions (2) and (5) give

$$
\left(2^{\prime}\right) \frac{\partial E_{y}}{\partial y}=-\frac{\partial E_{x}}{\partial x}
$$

This shows that $E_{y}$ determines $E_{x}$ up to an arbitrary function of $x$ only. But since $E_{x}$ and $E_{y}$ satisfy the reduced wave equation $\Delta u+k^{2} u=0$, the same must be true for both sides of $\left(2^{\prime}\right)$. Furthermore, from $(6)$ and $(7)$ we know that $E_{x}$ must approach 0 at $\infty$. This fact gives us the uniqueness we seek. For if $\left(2^{\prime}\right)$, with a fixed $E_{y}$, gave rise to two different solutions for $E_{x}$, then their difference could only be a function of $x$ alone. If this difference is $D(x)$, then

$$
\frac{d^{2}}{d x^{2}} D(x)+k^{2} D(x)=0
$$

and

$$
D(x) \rightarrow 0 \text { at } \infty .
$$

This implies $D(x) \equiv 0$. Thus, in what follows, we shall limit our attention to the determination of the $y$-component of the electric field. Our first task is to write the boundary conditions on $E_{y}$.

From (1) we have

(a) $\Delta E_{y}+k^{2} E_{y}=0$.

From (3) we have
(b) $\frac{\partial E_{y}}{\partial y}(x, \pm q \pi)=0 \quad x>0$
(c) $\frac{\partial E_{y}}{\partial y}(x, \pm \pi)=0 \quad x<0$
(d) $E_{y}(o, y)=0 \quad q \pi<|y|<\pi$.

Note that in (b) and (c) we used the fact that $E_{x}=0$ everywhere on those boundaries. This implied that $\frac{\partial E_{x}}{\partial x}=0$, there; and then $\left(2^{\prime}\right)$ was used to get (b) and (c). Continuity conditions in the aperture are
(e) $E_{y}\left(0^{+}, y\right)=E_{y}\left(0^{-}, y\right)$
$|y|<q \pi$
(f) $\frac{\partial E_{y}}{\partial x}\left(0^{+}, y\right)=\frac{\partial E_{y}}{\partial x}\left(0^{-}, y\right)$
$|y|<q \pi$.

And from (6) and (7)
(g) $\lim _{x \rightarrow-\infty}\left|E_{y}-\tau E_{o} e^{i k x}\right|=0$
(h) $\lim _{x \rightarrow+\infty}\left|E_{y}-E_{o} e^{i k x}-\rho E_{o} e^{-i k x}\right|=0$.

\section{Method and Summary}

The problem described above has been treated before, and the material has been presented by Marcuvitz [1951, p. 141] and by Saxon [1943]. Quantitative data have been given by Marcuvitz [1951, p. 307]. Furthermore, the problem has been treated earlier as a perturbation of the electrostatic case [Marcuvitz, 1951, p. 153], a method which will be used as the starting point for the present paper also.

The essential feature of the present approach is the explicit inversion of an infinite matrix which characterizes the electrostatic case. In the special case where $q=1 / 2$, this has already been done by Magnus and Oberhettinger [1950], who used algebraic relations connecting the matrix elements for this purpose. The present paper uses instead, integration in the complex plane, an approach which is of much wider applicability. The details may be described as follows:

The first step toward finding the reflection coefficient at the interface is to expand the field in each of the regions (I and II) in Fourier series. The modes are determined on each side by applying all the boundary conditions except the matching condition at the interface. The solution is then assumed to be a series in these modes with constant coefficients.

Applying the matching conditions at the interface gives rise to two infinite sets of linear equations, one arising from matching the fields, and the other from matching the normal derivatives. Using these two sets of equations we can eliminate one group of coefficients, leaving an infinite matrix equation for the other group and a scalar side condition involving the reflection coefficient. Solving this matrix equation, then, will allow us to apply the scalar side condition, and this will yield the value of the reflection coefficient.

The matrix equation which arises from the matching conditions is derived in full and is valid for all values of the wave number, $k<1$. The coefficients of the expansion of the field in region II give the unknown vector. The equation is solved using a perturbation method. The electrostatic case $(k=0)$ serves as the unperturbed case. We shall see that the ability to solve the electrostatic case for arbitrary right-hand sides provides us with enough power to determine the higher order terms in the electromagnetic case $(k \neq 0)$. Using this fact, and the scalar side condition, we shall determine the reflection coefficient up to terms of order $k^{2}$.

The one remaining facet of the method is the proof of solvability of the electrostatic case for arbitrary right-hand sides. Since the equation in this case is Laplace's equation, we may use conformal mapping. as an aid. We map the whole waveguide onto the infinite strip, using the Schwartz-Christoffel formula. This enables us to make use of the fact that each unknown is a Fourier coefficient which can be written as an integral (across the aperture) of the field. The field is written as the derivative of a harmonic potential. In this way the matrix equation may be solved in the electrostatic case for a specific right- 
hand side. However, this same technique can be used for more general right-hand sides. In order to accomplish this, we introduce into the original problem "unrealistic" modes, i.e., modes which do not die out at $+\infty$. The $N$ th unrealistic mode will give rise to a right-hand side consisting of $N+1$ nonzero entries. Thus, solving for all such unrealistic fields will yield a solution matrix whose product with the original matrix is a triangular matrix. The entries in the triangular matrix can be computed, using the conformal mapping; thus, the original matrix can be inverted. Therefore, we shall have solved the electrostatic case for arbitrary right-hand sides.

\section{Infinite System of Linear Equations}

In all that follows we shall use the notation that the restriction of $E_{y}$ to region $\mathrm{I}$ is denoted by $E_{y}^{\mathrm{I}}$ and similarly for $E_{y}^{\mathrm{II}}$. When referring to the entire waveguide we shall write simply $E_{y}$. Inasmuch as our ultimate goal is to apply a perturbation technique to this problem, we shall assume that $k<1$ so that

$$
n^{2}-k^{2} q^{2}>0 \text { for all integers } n \neq 0 .
$$

By separating variables in (a) and applying conditions (b) and (h) in region I we have

$$
E_{y}^{\mathrm{I}}(x, y)=E_{o}\left[e^{i k x}+\rho e^{-i k x}\right]+\sum_{n=1}^{\infty} c_{n} e^{i \frac{k_{n}}{q} x} \cos \frac{n}{q} y
$$

where

$$
k_{n}=i \sqrt{n^{2}-k^{2} q^{2}}, \quad n=1,2, \ldots
$$

In region II we have the additional condition (d) placed on the modes. So we must introduce a set of functions, $\phi_{N}$, satisfying (a), (c), (d), and (g). In addition, let the $N$ th function satisfy

$$
\left(\mathrm{e}^{\prime}\right) \phi_{N}\left(0^{-}, y\right)=\cos \frac{N}{q} y, \quad|y|<q \pi
$$

in the aperture. The reason for condition $\left(\mathrm{e}^{\prime}\right)$ is that the wave coming in from the right is a superposition of cosines in the aperture.

It will be necessary for us to have the $\phi_{N}$ explicitly, so we shall derive them here. The $N$ th mode must satisfy the conditions indicated in the sketch below.

Separating variables, we see that $\phi_{N}$ can be expanded in a series of the form

$$
\phi_{N}(x, y)=\sum_{n=0}^{\infty} \alpha_{n}^{(N)} e^{i l_{n} x} \cos n y \quad \text { (region II) }
$$

where

$$
l_{n}= \begin{cases}-i \sqrt{n^{2}-k^{2}} & n \neq 0 \\ k & n=0 .\end{cases}
$$

At $x=0$ we have

$\phi_{N}(o, y)=\sum_{n=0}^{\infty} \alpha_{n}^{(N)} \cos n y= \begin{cases}\cos \frac{N}{q} y & |y|<q \pi \\ 0 & q \pi<|y|<\pi .\end{cases}$

Consider the case $N \neq 0$, and multiply both sides by $\cos m y$ and integrate

$$
\begin{aligned}
& \alpha_{o}^{(N)}=\frac{1}{\pi} \int_{0}^{q \pi} \cos \frac{N}{q} y d y=0 \\
& \alpha_{m}^{(N)}=\frac{2}{\pi} \int_{0}^{q \pi} \cos m y \cos \frac{N}{q} y d y .
\end{aligned}
$$

Thus

$$
\phi_{N}(x, y)=\sum_{m=1}^{\infty} S_{m N} e^{i l_{m} x} \cos m y \quad N=1,2, \ldots
$$

where, for $m=1,2,3, \ldots$ and $N=0,1,2, \ldots$

$S_{m N}=\frac{2}{\pi} \int_{0}^{q \pi} \cos m y \cos \frac{N}{q} y d y=(-1)^{N} \frac{2 m \sin (q m \pi)}{\pi\left(m^{2}-N^{2} / q^{2}\right)}$.

Similarly, for $N=0$, we get

$$
\phi_{o}(x, y)=q e^{i k x}+\sum_{m=1}^{\infty} S_{m o} e^{i l_{m} x} \cos m y .
$$

The field in region II may now be written

$$
E_{y}^{\mathrm{II}}(x, y)=\sum_{n=0}^{\infty} d_{n} \phi_{n}(x, y) .
$$

Applying condition (e), we have

$$
E_{o}(1+\rho)+\sum_{n=1}^{\infty} c_{n} \cos \frac{n}{q} y=\sum_{m=0}^{\infty} d_{m} \phi_{m}(o, y) \quad|y|<q \pi
$$

but, by definition,

$$
\phi_{m}(o, y)=\cos \frac{m}{q} y \quad|y|<q \pi .
$$

Therefore,

$$
\begin{aligned}
E_{o}(1+\rho) & =d_{0} \\
c_{n} & =d_{n} \quad n=1,2, \ldots .
\end{aligned}
$$

Differentiate (1) with respect to $\mathrm{x}$

$$
\frac{\partial E_{y}^{1}(x, y)}{\partial x}=i k E_{o}\left[e^{i k x}-\rho e^{-i k x}\right]+\frac{i}{q} \sum_{n=1}^{\infty} c_{n} k_{n} e^{i \frac{k_{n}}{q} x} \cos \frac{n}{q} y .
$$

Let

$$
\psi_{m}(x, y)=\frac{\partial \phi_{m}(x, y)}{\partial x}
$$

Condition (f) gives 


$$
i k E_{o}(1-\rho)+\frac{i}{q} \sum_{n=1}^{\infty} c_{n} k_{n} \cos \frac{n}{q} y=\sum_{m=0}^{\infty} d_{m} \psi_{m}(o, y) .
$$

Solving for the Fourier coefficients on the left-hand side,

$$
\begin{gathered}
i k E_{o}(1-\rho)=\frac{1}{q \pi} \int_{0}^{q \pi} \sum_{m=0}^{\infty} d_{m} \psi_{m}(o, y) d y \\
c_{n}=\frac{2}{\pi k_{n} i} \int_{o}^{q \pi} \sum_{m=0}^{\infty} d_{m} \psi_{m}(o, y) \cos \frac{n}{q} y d y \quad n=1,2, \ldots
\end{gathered}
$$

Combining (5) with (7),

$$
d_{n}=\frac{2}{\pi k_{n} i} \int_{0}^{q \pi} \sum_{m=0}^{\infty} d_{m} \psi_{m}(o, y) \cos \frac{n}{q} y d y \quad n=1,2, \ldots
$$

From (2) and (3) it can be seen that

$$
\begin{aligned}
& \psi_{o}(x, y)=i k q e^{i k x}+i \sum_{r=1}^{\infty} S_{r o} l_{r} e^{i l_{r} x} \cos r y \\
& \psi_{m}(x, y)=i \sum_{r=1}^{\infty} l_{r} S_{r m} e^{i l_{r} x} \cos r y \quad m=1,2, \ldots
\end{aligned}
$$

Therefore,

$$
\sum_{m=0}^{\infty} d_{m} \psi_{m}(o, y)=i k q d_{o}+i \sum_{m=0}^{\infty} d_{m} \sum_{r=1}^{\infty} S_{r m} l_{r} \cos r y .
$$

Substituting into (8),

$d_{n}=\frac{2}{\pi k_{n}} \int_{0}^{p_{\pi}}\left\{k q d_{o}+\sum_{m=0}^{\infty} d_{m} \sum_{r=1}^{\infty} l_{r} S_{r m} \cos r y\right\} \cos \frac{n}{q} y d y$ $n=1,2, \ldots$

$d_{n}=\sum_{m=0}^{\infty} d_{m} \sum_{r=1}^{\infty} \frac{l_{r}}{k_{n}} S_{r m} S_{r n} \quad n=1,2, \ldots$

Equation (11) is an infinite system of linear equations in the unknown Fourier coefficients, $d_{n}$.

A scalar side condition is obtained by substituting (10) into (6).

$$
\begin{aligned}
i k E_{o}(1-\rho) & =\frac{1}{q \pi} \int_{0}^{q \pi}\left\{i k q d_{o}+i \sum_{m=0}^{\infty} d_{m} \sum_{r=1}^{\infty} S_{r m} l_{r} \cos r y\right\} d y \\
& =i k q d_{o}+\frac{i}{q \pi} \sum_{m=0}^{\infty} d_{m} \sum_{r=1}^{\infty} \frac{1}{r} S_{r m} l_{r} \sin r \pi q .
\end{aligned}
$$

It can be seen from $(3),(5),(4)$ and condition $(\mathrm{g})$ that the transmission coefficient, $\tau$, is given by

$$
\tau=q(1+\rho),
$$

which gives a simple relation between the reflection and transmission coefficient. Using the value of $d_{o}$ found in (5), we have from (12)

$$
\sum_{m=0}^{\infty} d_{m} \sum_{r=1}^{\infty} \frac{l_{r}}{q \pi r} S_{r m} \sin q \pi r=k E_{o}(1-\rho-q-\rho q) .
$$

This scalar condition (together with (5)) will be used to evaluate $\rho$ after (11) has been solved for the $d_{n}$ 's.

\section{Perturbation Method}

In order to use the perturbation method in the solution of the equations of the previous section, we must establish their explicit dependence upon $k$. Having done that, we shall solve the electrostatic problem $(k=0)$ explicitly and use it as the unperturbed case.

Equation (11) depends upon $k$ only in its term, $\frac{l_{r}}{k_{n}}$.

$$
\begin{aligned}
\frac{l_{r}}{k_{n}}=-\frac{\sqrt{r^{2}-k^{2}}}{\sqrt{n^{2}-k^{2} q^{2}}}=-\frac{r}{n} & \frac{\sqrt{1-\frac{k^{2}}{r^{2}}}}{\sqrt{1-\frac{k^{2} q^{2}}{n^{2}}}} \\
& =-\frac{r}{n} \sqrt{\left(1-\frac{k^{2}}{r^{2}}\right)\left(1+\frac{k^{2} q^{2}}{n^{2}}+\ldots\right)}
\end{aligned}
$$

$$
\begin{aligned}
& =-\frac{r}{n} \sqrt{1-\left(\frac{k^{2}}{r^{2}}-\frac{k^{2} q^{2}}{n^{2}}\right)+\ldots} \\
& =-\frac{r}{n}+\frac{r}{2 n}\left(\frac{k^{2}}{r^{2}}-\frac{k^{2} q^{2}}{n^{2}}\right)+\ldots \\
& =-\frac{r}{n}+\frac{k^{2}}{2}\left(\frac{1}{n r}-\frac{q^{2} r}{n^{3}}\right)+\ldots
\end{aligned}
$$

Equation (14) depends upon $k$ only in its term, $l_{r}$.

$$
l_{r}=-i \sqrt{r^{2}-k^{2}}=-i r \sqrt{1-\frac{k^{2}}{r^{2}}}=-i r+\frac{i k^{2}}{2 r}+\ldots
$$

Neglecting all terms involving powers of $k$ higher than the second, eq (11) can be written in matrix notation as follows: let

$$
\vec{d}=\left(d_{0}, d_{1}, d_{2}, \ldots\right)
$$

$T=$ the matrix of elements $T_{n r}=\frac{r}{n} S_{r n}, \quad n \neq 0$,

$$
=0, \quad n=0,
$$

$S=$ matrix of elements $S_{r n}=\frac{2}{\pi} \int_{0}^{q \pi} \cos r y \cos \frac{n}{q} y d y$

(as previously deiined),

$U=$ matrix of elements 


$$
\begin{aligned}
U_{n r} & =-\frac{1}{2}\left(\frac{1}{r^{2}}-\frac{q^{2}}{n^{2}}\right) T_{n r} \quad n, r \neq 0, \\
& =0 \quad n \text { or } r=0 .
\end{aligned}
$$

Now we add (5) to (11) and use the notation that $\overrightarrow{1}$ is the vector

$$
\overrightarrow{1}=\{1,0,0,0, \ldots\} .
$$

We assume that all summations go from 0 to $+\infty$.

$$
\vec{d}=-\left\{\left(T+k^{2} U\right) S\right\}(\vec{d})+E_{o}(1+\rho) \overrightarrow{1} .
$$

If $\mathrm{I}$ is the identity matrix, we have

$$
\left\{\mathrm{I}+T S+k^{2} U S\right\} \overrightarrow{(d)}=E_{o}(1+\rho) \overrightarrow{1} .
$$

Let

$$
\begin{aligned}
& T_{r m}^{*}=-\frac{i}{q \pi} S_{r m} \sin q \pi r \quad r=0,1,2, \ldots \\
& U_{r m}^{*}=-\frac{1}{2 r^{2}} T_{r m}^{*} \quad r=1,2, \ldots \\
& U_{o m}^{*}=0 .
\end{aligned}
$$

Then (14) becomes

$$
\sum_{m=0}^{\infty} d_{m} \sum_{r=0}^{\infty}\left(T_{r m}^{*}+k^{2} U_{r m}^{*}\right)=k E_{o}(1-\rho-q-\rho q)
$$

For shorthand, let us define vectors $\vec{t}^{*}$ and $\vec{u}^{*}$ whose components are:

$$
\begin{aligned}
& t_{m}^{*}=\sum_{r=0}^{\infty} T_{r m}^{*} \\
& u_{m}^{*}=\sum_{r=0}^{\infty} U_{r m}^{*} .
\end{aligned}
$$

Then (17) can be rewritten as

$$
\left(t^{*}+k^{2} \vec{u}^{*}\right) \cdot \vec{d}=k E_{o}(1-\rho-q-\rho q) .
$$

Equations (16) and (18) form the system which we shall solve. First we shall solve (16) using the perturbation method to invert the matrix. Then we shall use the solution vector, $\vec{d}$, in (18) to compute the reflection coefficient, $\rho$.

The method will proceed as follows. We shall show that the electrostatic system

$$
\{\mathrm{I}+T S\} \overrightarrow{(d)}=\vec{R}
$$

can be solved for arbitrary right-hand sides, $\vec{R}$, in such a way that $\overrightarrow{t^{*}} \cdot \vec{d}=0$. When this is known, the full system can be solved up to terms of order $k^{2}$ by setting

$$
\vec{d}=\vec{e}+k^{2} \vec{f}
$$

Equation (16) becomes

$$
\left\{I+T S+k^{2} U S\right\}\left(\vec{e}+k^{2} \vec{f}\right)=E_{o}(1+\rho) \overrightarrow{1}
$$

Thus, neglecting powers of $k$ higher than the second, $\{\mathrm{I}+T S\}(\vec{e})+k^{2}\{\mathrm{I}+T S\}(\vec{f})+k^{2}\{U S\} \overrightarrow{(e)}=E_{o}(1+\rho) \overrightarrow{1}$

We now can find a unique vector, $\vec{e}$, such that

$$
\{\mathrm{I}+T S\} \overrightarrow{(e)}=\overrightarrow{1}
$$

(which implies that $E_{o}(1+\rho) \vec{e}$ solves the electro-

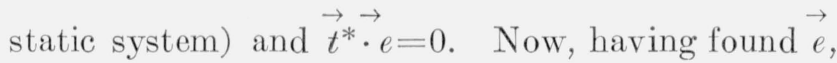
we compute $\{U S\} \overrightarrow{(e)}$ and solve

$$
\{I+T S\}(\vec{f})=-\{U S\} \overrightarrow{(e)}
$$

in such a way that $\vec{t}^{*} \cdot \vec{f}=0$.

Having found $\vec{d}\left(=\vec{e}+k^{2} f\right)$, we substitute this solution vector into (18).

$$
\left(t^{*}+k^{2} \vec{u}^{*}\right) \cdot\left(\vec{e}+k^{2} \vec{f}\right)=\frac{k E_{o}(1-\rho-q-\rho q)}{E_{o}(1+\rho)} .
$$

But $\vec{t}^{*} \cdot \vec{e}=\overrightarrow{t^{*}} \cdot \vec{f}=0$.

Therefore,

$$
\begin{aligned}
k^{2} \vec{u}^{*} \cdot \vec{e} & =\frac{k E_{o}(1-\rho-q-\rho q)}{E_{o}(1+\rho)} \\
\rho & =\frac{1-\left(q+k \vec{u}^{*} \cdot \vec{e}\right)}{1+\left(q+k \vec{u}^{*} \cdot \vec{e}\right)} .
\end{aligned}
$$

Our main result may now be stated as

Theorem 1. For sufficiently small values of $k$, the reflection coefficient $\rho$ is given by formula (19), where the vector $\vec{u}^{*}$ has been defined by equation (17) and where the components of the vector $\vec{e}$ are defined by equations (38) and (39); the vector $\vec{e}$ itself describes the solution of the electrostatic problem $(k=0)$. 


\section{Electrostatic Case}

We bave the system of linear equations

$$
\{\mathrm{I}+T S\}(\vec{d})=\vec{R}
$$

and we wish to show that it can be solved for arbitrary right-hand sides in such a way that the solution vector, $\vec{d}$, satisfies the scalar condition

$$
\overrightarrow{t^{*}} \cdot \vec{d}=0
$$

To this end we first invert the matrix $\{I+T S\}$. The method we shall use is to produce a matrix, $D$, and a triangular matrix, $\Delta$, such that

$$
\{\mathrm{I}+T S\}\{D\}=\Delta .
$$

Since $\Delta$ is triangular, its inverse can easily be computed; thus, the full solution is

$$
\{\mathrm{I}+T S\}^{-1}=D \Delta^{-1} .
$$

First we shall construct the triangular matrix, $\Delta$. To this end, let us consider the field in region I. It has the form

$$
E_{y}^{\mathrm{I}}(x, y)=\sum_{n=0}^{\infty} c_{n} e^{-\frac{n}{q} x} \cos \frac{n}{q} y
$$

in the electrostatic case. If we relax the condition at $+\infty$ and allow the field to become exponentially large, new modes may be introduced. We shall refer to these as "unrealistic" modes. Let the $N$ th unrealistic mode be denoted by $E_{N}^{\mathrm{I}}(x, y)$. It will increase exponentially for $x \rightarrow \infty$, and the order of magnitude of the function at $x \rightarrow \infty$ is determined by the first term $(n=-N)$ in the expansion:

$$
E_{N}^{\mathrm{I}}(x, y)=\sum_{n=-N^{Y}}^{\infty} c_{n}^{N} e^{-\frac{n}{q} x} \cos \frac{n}{q} y .
$$

We wish to determine $E_{N}^{\mathrm{r}}$ in such a manner that it satisfies all conditions for $E_{y}$ except for (7). This implies that, in region II, we have

$$
E_{N}^{\mathrm{II}}(x, y)=\sum_{n=0}^{\infty} d_{n}^{N} \phi_{n}(x, y)
$$

where, in the electrostatic case,

$$
\begin{aligned}
& \phi_{0}(x, y)=q+\sum_{m=1}^{\infty} S_{m o} e^{m x} \cos m y \\
& \phi_{n}(x, y)=\sum_{m=1}^{\infty} S_{m n} e^{m x} \cos m y \quad n \neq 0 \\
& \psi_{n}(x, y)=\frac{\partial \phi_{n}}{\partial x}=\sum_{m=1}^{\infty} m S_{m n} e^{m x} \cos m y \quad \text { all } n .
\end{aligned}
$$

Equations (21) and (22) provide us with an infinitude of new fields. Solution of these will yield the matrices, $D$ and $\Delta$, which we seek.

Matching (21) and (22) in the aperture $x=0$, $|y|<q \pi$,

$$
\sum_{n=-N}^{\infty} c_{n}^{N} \cos \frac{n}{q} y=\sum_{n=0}^{\infty} d_{n}^{N} \phi_{n}(o, y)
$$

This implies that

$$
\begin{array}{rlrl}
c_{n}^{N} & =d_{n}^{N} & & n>N \\
c_{n}^{N}+c_{-n}^{N}=d_{n}^{N} & & 0<n<N \\
c_{o}^{N} & =d_{o}^{N}, & &
\end{array}
$$

since

$$
\cos \frac{n}{q} y=\cos \left(-\frac{n}{q}\right) y
$$

and

$$
\phi_{n}(o, y)=\cos \frac{n}{q} y, \quad|y|<q \pi .
$$

Let us define

Then

$$
\begin{aligned}
& c_{n}^{+}= \begin{cases}c_{n}^{N} & n>0 \\
\frac{1}{2} c_{o}^{N} & n=0\end{cases} \\
& c_{n}^{-}= \begin{cases}0 & n>N \\
c_{-n}^{N} & 0<n \leq N \\
\frac{1}{2} c_{o}^{N} & n=0 .\end{cases}
\end{aligned}
$$

$$
d_{n}^{N}=c_{n}^{+}+c_{n}^{-}, \text {for all } n .
$$

We apply the derivative condition

$$
\begin{gathered}
\frac{\partial E_{N}^{\mathrm{I}}(x, y)}{\partial x}=-\frac{1}{q} \sum_{m=-N}^{\infty} m c_{m}^{N} e^{-\frac{m}{q} x} \cos \frac{m}{q} y \\
\frac{\partial E_{N}^{\mathrm{II}}(x, y)}{\partial x}=\sum_{n=0}^{\infty} d_{n}^{N} \psi_{n}(x, y) \\
\sum_{m=1}^{\infty} \frac{m}{q}\left(c_{m}^{-}-c_{m}^{+}\right) \cos \frac{m}{q} y=\sum_{n=0}^{\infty} d_{n}^{N} \psi_{n}(o, y) \quad|y|<q \pi .
\end{gathered}
$$

Multiply by $\cos \frac{n}{q} y$ and integrate

$$
\frac{n}{q}\left(c_{n}^{-}-c_{n}^{+}\right)=\frac{2}{q \pi} \int_{0}^{q \pi} \sum_{m=0}^{\infty} d_{m}^{N} \psi_{m}(0, y) \cos \frac{n}{q} y d y .
$$

Multiply this by $\frac{q}{n}$ and add (25)

$$
2 c_{n}^{-}=d_{n}^{N}+\sum_{m=0}^{\infty} d_{m}^{N} \frac{2}{\pi n} \int_{0}^{q_{\pi}} \psi_{m}(o, y) \cos \frac{n}{q} y d y .
$$

However, it is easily seen that (27) is merely 


$$
\{\mathrm{I}+T S\}\left(\vec{d}_{N}\right)=2{\overrightarrow{c_{N}^{-}}}^{-}
$$

where

$$
\vec{d}_{N}=\left(d_{o}^{N}, d_{1}^{N}, \ldots\right)
$$

and

$$
\vec{c}_{N}^{-}=\left(\frac{1}{2} c_{o}^{N}, c_{-1}^{N}, \ldots, c_{-N}^{N}, 0,0 \ldots\right) .
$$

If for each $N$ we can compute $\vec{c}_{N}^{-}$and $\vec{d}_{N}$, we will have found the matrices $D$ and $\Delta$. The solution vectors, $\vec{d}_{N}$, will comprise $D$; and the right-hand sides, $\overrightarrow{c_{N}^{-}}$, will comprise the triangular matrix, $\Delta$.

Thus, we must do the following for each $N$ :

(i) Compute the vector $\overrightarrow{c_{N}^{-}}$.

(ii) Find the solution vector $\vec{d}_{N}$.

Problem (i) may be solved as follows: We map the waveguide structure onto the strip $|v| \leq \pi$ in the $w=u+i v$ plane. The Schwartz-Christoffel formula gives us the mapping implicitly. We may state the result as

Lemma 1. The mapping of the interior of the waveguide in the $z=(x+i y)$ plane (as drawn in fig. 1) onto the strip $|v| \leqq \pi$ of the $w=(u+i v)$ plane is given by

$$
z=\log \left[\frac{\left(\sqrt{q^{2} e^{w}+1}+q \sqrt{e^{w}+1}\right)^{2 q} e^{w}\left(1-q^{2}\right)^{1-q}}{\left(\sqrt{q^{2} e^{w}+1}+\sqrt{e^{w}+1}\right)^{2}}\right]
$$

$$
\frac{d w}{d z}=\sqrt{\frac{e^{w}+1}{q^{2} e^{w}+1}}
$$

where the square roots are determined by $\arg \left(q^{2} e^{w}+1\right)^{\frac{1}{2}}=0 \quad$ for $w=i \pi+2 \log q^{-1}+\sigma$ and $\sigma \leqq 0$,

$\arg \left(q^{2} e^{w}+1\right)^{\frac{1}{2}}=\frac{\pi}{2} \quad$ for $w=i \pi+2 \log q^{-1}+\sigma$ and $\sigma>0$,

$\arg \left(e^{w}+1\right)^{\frac{1}{2}}=0 \quad$ for $w=i \pi+\tau, \tau \leqq 0$,

$\arg \left(e^{w}+1\right)^{\frac{1}{2}}=\frac{\pi}{2} \quad$ for $w=i \pi+\tau, \tau>0$.

The following points correspond to each other:

$$
\begin{array}{ll}
w=i \pi-\infty & \text { and } z=i \pi-\infty \\
w=i \pi & \text { and } z=i \pi \\
w=i \pi+2 \log q^{-1} & \text { and } z=i \pi q \\
w=i \pi+\infty & \text { and } z=i \pi q+\infty .
\end{array}
$$

Let $\zeta=e^{-\frac{z}{q}}, \sigma=e^{-W}$. Substituting into (29),

$$
\zeta=\sigma \frac{\left(\sqrt{q^{2}+\sigma}+\sqrt{1+\sigma}\right)^{\frac{2}{q}}}{\left(\sqrt{q^{2}+\sigma}+q \sqrt{1+\sigma}\right)^{2}\left(1-q^{2}\right)^{\frac{1-q}{q}}} .
$$

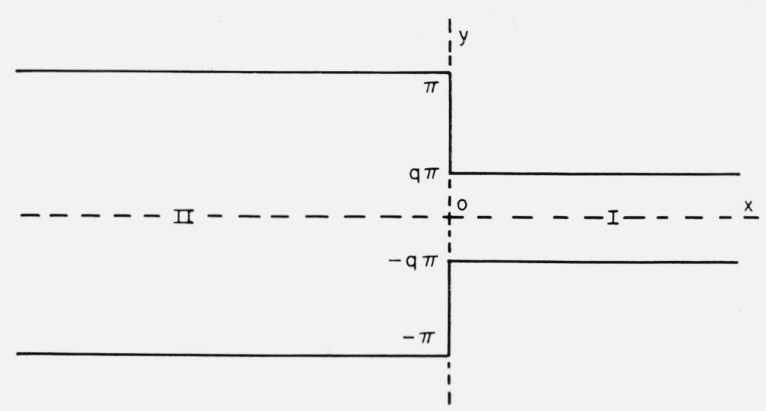

Figure 1.

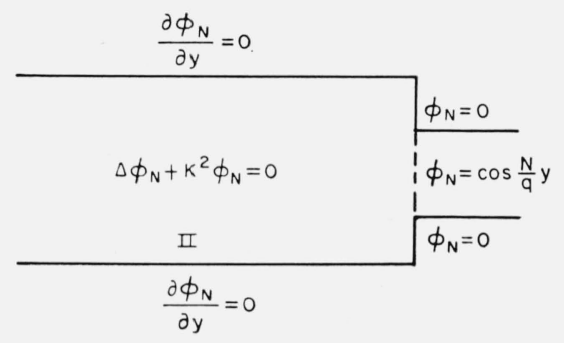

Figure 2.

Equation (30) shows that $\sigma$ can be expanded in a power series in $\zeta$. The field $E_{N}$ can be written as the real part of the derivative of the potential, $e^{N w}$.

Let $\Phi_{N}=\frac{d}{d z} e^{N w}$ so that $E_{N}=\operatorname{Re} \Phi_{N}$. We assert that $\Phi_{N}$ can be expanded in a series of the type

$$
\begin{aligned}
c_{-N}^{(N)} e^{\frac{N z}{q}}+c_{1-N}^{(N)} e^{\frac{(1-N)}{q} z}+\ldots+ & c_{o}^{(N)} \\
& +\sum_{l=1}^{\infty} c_{l}^{(N)} e^{-\frac{l z}{q}}=\sum_{l=-N}^{\infty} c_{l}^{(N)} \zeta^{l .}
\end{aligned}
$$

This form follows from (30) and from

$$
\Phi_{N}=N e^{N w} \frac{d w}{d z}=N \sigma^{-N} \sqrt{\frac{1+\sigma}{q^{2}+\sigma}} .
$$

The residue theorem asserts that

$$
c_{-l}^{(N)}=\frac{1}{2 \pi i} \oint_{\zeta=0} \Phi_{N} \zeta^{l-1} d \zeta \quad 1 \leqq l \leqq N
$$

This reduces to the expression (31) for $C_{-l}^{(N)}$ as an integral over a small circle around $\sigma=0$, taken in the positive sense.

$$
\begin{aligned}
c_{-l}^{(N)}=\frac{1}{2 \pi i} \frac{N}{q\left(1-q^{2}\right)^{(1-q)} \frac{l}{q}} \oint_{\sigma=0} \sigma^{l-N-1} & \\
& \frac{\left(\sqrt{q^{2}+\sigma}+\sqrt{1+\sigma}\right)^{2 \frac{l}{q}}}{\left(\sqrt{q^{2}+\sigma}+q \sqrt{1+\sigma}\right)^{2 l}} d \sigma .
\end{aligned}
$$


This verifies that $c_{-l}^{(N)}=0, l>N$.

Problem (ii) may be solved by using the fact that in the aperture each component of the vector $\vec{d}_{N}$ is a coefficient of a Fourier series in the aperture.

But

$$
d_{m}^{N}=\frac{1}{q \pi} \int_{-q \pi}^{q \pi} E_{N} \cos \frac{m}{q} y d y .
$$

$$
E_{N}=\operatorname{Re} \Phi_{N}=\operatorname{Re} N e^{N w} \frac{d w}{d z}
$$

Writing the full complex integral (and dropping the "Re" for now),

$$
\begin{aligned}
d_{m}^{N} & =\frac{1}{2 \pi q i} \int_{-q \pi i}^{q \pi i} N e^{N w} \frac{d w}{d z}\left\{e^{\frac{m}{q} z}+e^{-\frac{m}{q} z}\right\} d z \\
& =\frac{N}{2 \pi q i} \int_{-\pi i+\log \left(\frac{1}{q^{2}}\right)}^{\pi i+\log \left(\frac{1}{q^{2}}\right)} e^{N w}\left\{e^{\frac{m}{q} F(w)}+e^{-\frac{m}{q} F(w)}\right\} d w,
\end{aligned}
$$

where $F(w)$ is the Schwartz-Christoffel mapping given in (29). Using the substitution $\lambda=e^{x}$

$$
\begin{array}{r}
d_{m}^{N=\operatorname{Re}} \frac{N}{2 \pi q i}\left[\left(1-q^{2}\right)^{(1-q) \frac{m}{q}} \int \lambda^{N-1+\frac{m}{q}}\right. \\
\frac{\left(\sqrt{q^{2} \lambda+1}+q \sqrt{\lambda+1}\right)^{2 m}}{\left(\sqrt{q^{2} \lambda+1}+\sqrt{\lambda+1}\right)^{2 \frac{m}{q}}} d \lambda+\left(1-q^{2}\right)^{(q-1) \frac{m}{q}} \\
\left.\int \lambda^{N-1-\frac{m}{q}} \frac{\left(\sqrt{q^{2} \lambda+1}+\sqrt{\lambda+1}\right)^{\frac{2 m}{q}}}{\left(\sqrt{q^{2} \lambda+1}+q \sqrt{\lambda+1}\right)^{2 m}} d \lambda\right]
\end{array}
$$

The path of integration is around a circle of radius $\frac{1}{q^{2}}$ in the positive sense, as illustrated in figure 3.

It remains now to show that solutions of the system

satisfy

$$
\{I+T S\}(\vec{d})=\vec{R}
$$

$$
\vec{t}^{*} \cdot \vec{d}=0
$$

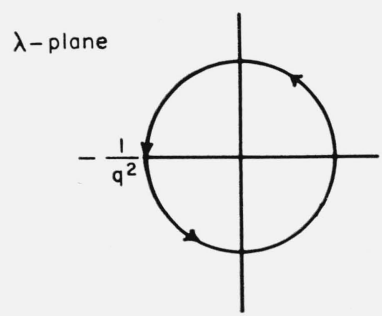

Figure 3.
The reason for this is that the components $d_{m}$ of $\vec{d}$ as well as the components of all the vectors $\vec{d}_{N}$ are the Fourier coefficients of the derivative of a potential function $\operatorname{Re} \Phi=\frac{\partial v}{\partial y}$ (or, in general, of $\operatorname{Re} \Phi_{N}=\frac{\partial}{\partial y}$ [sin $N v \exp N u]$ ). In fact, the definition of the $t_{m}^{*}$ by (17b) shows that

$$
t_{m}^{*}=\int_{-q \pi}^{q \pi} \psi_{m}(x, y) d y
$$

where the $\psi_{m}(x, y)$ are defined in the electrostatic case by $(9 a),(9 b)$ with the additional condition $k=0$. Now the $d_{m}$ are derived by the fact that

$$
\Sigma d_{m} \psi_{m}(x, y)
$$

is the expansion for $\operatorname{Re} \Phi$ (or, more generally, $\operatorname{Re} \Phi_{N}$ ) in region II. Therefore we have

$$
\overrightarrow{t^{*}} \cdot \vec{d}=\frac{1}{2 q \pi} \int_{-q \pi}^{q \pi} \frac{\partial^{2} v(o, y)}{\partial y \partial x} d y=\left.\frac{\partial v}{\partial x}\right|_{(o,-q \pi)} ^{(o, q \pi)} .
$$

But $v$ is constant on both boundaries, so that there $\partial v / \partial x=0$. Therefore, $\vec{t}^{*} \cdot \vec{d}=0$.

\section{Convergence}

It has been tacitly assumed in the previous computations that the convergence of the various inînite series is good enough to justify the operations performed. For example, the final formula for the reflection coefficient (19) involves an infinite series

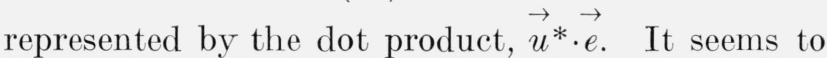
be a rather difficult task to prove that our perturbation method leads to a convergent procedure for the computation of $\rho$, at least for sufficiently small $k$. All we shall do here is this: We shall prove that the approximation formula of Theorem 1 is meaningful. For this purpose, we must prove:

Theorem 2: The infinite sum represented by the product $\vec{u}^{*} \cdot \vec{e}$ converges absolutely.

From equation (17) it can be seen that

$$
\begin{array}{r}
\overrightarrow{u^{*} \cdot \vec{e}}=\sum_{m=0}^{\infty} e_{m} \sum_{r=1}^{\infty} \frac{i}{2 q \pi r^{2}} \sin q \pi r \frac{2}{\pi} \int_{0}^{q_{\pi}} \cos r y \cos \frac{m}{q} y d y \\
=\frac{i}{q \pi^{2}} \sum_{m=0}^{\infty} e_{m}\left[\sum_{r=1}^{\infty} \frac{\sin q \pi r}{r^{2}} \int_{0}^{q_{\pi}} \cos r y \cos \frac{m}{q} y d y\right] .
\end{array}
$$

We shall show first that the factor in brackets is less than a constant times $1 / m$ for $m$ large. This may be seen as follows: The factor in question is the $m$ th Fourier coefficient of the function

$$
f(y)=\frac{1}{2} \sum_{r=1}^{\infty}\left\{\frac{\sin r(q \pi+y)}{r^{2}}+\frac{\sin r(q \pi-y)}{r^{2}}\right\} .
$$


The formula

$$
\sum_{r=1}^{\infty} \frac{\cos r y}{r}=-\log (2 \cos (y / 2))
$$

shows that $f(y)$ is differentiable except for isolated points in any inite interval. According to Whittaker-Watson [1958], sec. 9.3, the $m$ th Fourier coefficient of such a function has the order of magnitude of $1 / \mathrm{m}$.

In order to complete the proof of convergence of $\vec{u}^{*} \cdot \vec{e}$ in (35), we must demonstrate the absolute convergence of

$$
\sum_{m=1}^{\infty} \frac{1}{m} e_{m}
$$

Each component of the vector $\vec{e}$ is a coefficient of the Fourier expansion of the field in the aperture.

$$
e_{m}=\frac{1}{q \pi} \int_{-q \pi}^{q \pi} E_{y} \cos \frac{m}{q} y d y .
$$

Inasmuch as $E_{y}$ vanishes for $q \pi<|y|<\pi$, (37) can be written as

$$
\begin{aligned}
e_{m} & =\frac{1}{q \pi} \int_{-\pi}^{\pi} E_{y} \cos \frac{m}{q} y d y \\
& =\frac{1}{2 \pi q i} \int_{-\pi i}^{\pi i} \frac{d w}{d z}\left(e^{\frac{m}{q} z}+e^{-\frac{m}{q} z}\right) d z .
\end{aligned}
$$

We can convert this to a line integral in the $w$-plane using the Schwartz-Christoffel mapping given in (29).

$$
\begin{gathered}
e_{m}=\frac{1}{2 \pi q i}\left\{\left(1-q^{2}\right)^{(1-q) \frac{m}{q}} \int_{C} e^{\frac{m}{q} w} \frac{\left(\sqrt{q^{2} e^{w}+1}+q \sqrt{e^{w}+1}\right)^{2 m}}{\left(\sqrt{q^{2} e^{w}+1}+\sqrt{e^{w}+1}\right)^{\frac{2 m}{q}}} d w\right. \\
\left.+\frac{1}{\left(1-q^{2}\right)^{(1-q) \frac{m}{q}}} \int_{C} e^{-\frac{m}{q} w} \frac{\left(\sqrt{q^{2} e^{w}+1}+\sqrt{e^{w}+1}\right)^{\frac{2 m}{q}}}{\left(\sqrt{q^{2} e^{w}+1}+q \sqrt{e^{w}+1}\right)^{2 m}} d w\right\} .
\end{gathered}
$$

The path of integration, $C$, is illustrated in the following sketch (fig. 4 ):

The first integral will converge if the path is altered in such a way that $\operatorname{Re}(w)<0$, so we shall change the path into the one indicated by figure 5 . Along the bottom path we may put

$$
w=-\pi i-s, \quad 0 \leqq s<\infty .
$$

By rewriting the first integral in terms of $s$, and by using the inequalities

$$
\begin{aligned}
\left(1-q^{2}\right)^{\frac{1}{2}} \leqq\left(1-q^{2} e^{-s}\right)^{\frac{1}{2}}+q\left(1-e^{-s}\right)^{\frac{1}{2}} & \\
& \leqq\left(1-q^{2} e^{-s}\right)^{\frac{1}{2}}+\left(1-e^{-s}\right)^{\frac{1}{2}}
\end{aligned}
$$

we see easily that the resulting integral can be majorized by $1 / 2 \pi m$. The same analysis goes through on the upper path of integration, and this shows the absolute convergence of the first half of (36).

For the second integral in (39) we can break up the path as indicated in ifgure 6 .

We see readily that the integrals along $C_{2}$ and $C_{3}$ cancel each other out exactly. We are left, then, with integrals along $C_{1}$ and $C_{4}$. By putting

$$
W=\pi i+s, \quad 0 \leqq s \leqq \log q^{-2}
$$

on $C_{4}$, and using the substitutions

$\cos \alpha=\left(1+e^{-s}\right)^{\frac{1}{2}}\left(1-q^{2}\right)^{-\frac{1}{2}}$,

$$
\cos \beta=q\left(e^{s}-1\right)^{\frac{1}{2}}\left(1-q^{2}\right)^{-\frac{1}{2}}
$$

$$
\frac{d \alpha}{d s}=-\frac{1}{2} e^{-s}\left(e^{-s}-q^{2}\right)^{-\frac{1}{2}}\left(1-e^{-s}\right)^{-\frac{1}{2}}, \quad 0 \leqq \alpha \leqq \pi / 2
$$

$$
\begin{aligned}
& \frac{d \beta}{d s}=-\frac{q}{2} e^{s}\left(e^{s}-1\right)^{-\frac{1}{2}}\left(1-q^{2} e^{s}\right)^{-\frac{1}{2}}, \quad 0 \leqq \beta \leqq \pi / 2 \\
& \gamma=\frac{\alpha}{q}-\beta, \frac{d \alpha}{d s}=-\frac{1}{2} \tan \beta \quad 0 \leqq \gamma \leqq \frac{\pi}{2}\left(\frac{1}{q}-1\right)
\end{aligned}
$$

we ind that the second integral along $C_{4}$ has the value

$$
\frac{(-1)^{m+1}}{2 \pi i q} \int_{0}^{\log q^{-2}} e^{2 i m \gamma} d s .
$$

Applying a similar method to transform the second integral along $C_{1}$, we find, after combining the results, the single integral

$$
\frac{(-1)^{m+1}}{\pi q} \int_{0}^{\log q^{-2}} \sin (2 m \gamma) d s,
$$

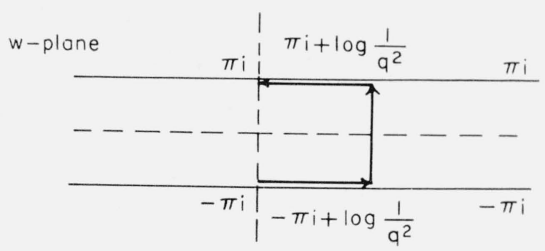

Figure 4.

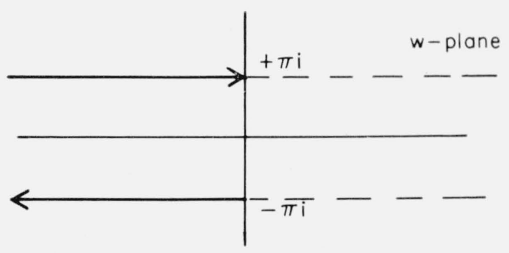

Figure 5. 


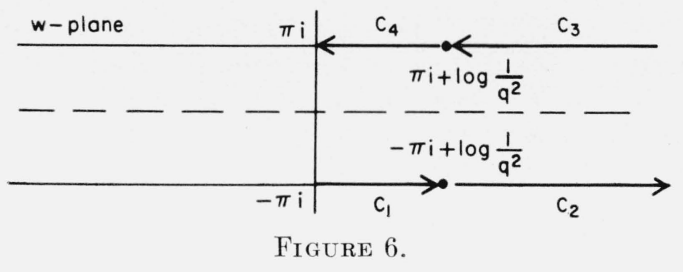

which is now our expression for the second integral in (39). It can be shown that (45) is majorized by a constant multiple of $m^{-2 / 3}$ for large values of $m$. The proof for this fact is somewhat tedious, and we shall give a sketch of it only.

Because of (43), the integral in (45) is singular at $s=\log q^{-2}, \beta=\pi / 2$, where $d s / d \gamma$ becomes infinite. To show that (45) exists and even becomes small for large $m$, we introduce the variable

$$
t=s-\log q^{-2}
$$

and show that $t$ depends analytically on $\gamma^{1 / 3}$ such that (with a constant $c$ )

$$
t=c^{2} \gamma^{2 / 3}+\text { higher terms in } \gamma^{1 / 3} .
$$

From (46), we can deduce that (45) behaves for large $m$ like

$$
\int_{0}^{\delta} \frac{\sin 2 m \gamma}{\gamma^{\frac{1}{3}}} d \gamma
$$

where $\delta$ is a fixed, sufficiently small real number. In turn, (47) can be shown to be of the order of $m^{-2 / 3}$ because of

$$
\int_{0}^{\infty} \frac{\sin 2 m \gamma}{\gamma^{\frac{1}{3}}} d \gamma=\Gamma\left(\frac{2}{3}\right)\left(\cos \frac{\pi}{6}\right)(2 m)^{-\frac{2}{3}}
$$

Combining all these results shows that $\vec{u} \cdot \vec{*} \cdot e$ converges absolutely, for we have shown that this sum behaves at worst like

$$
\sum_{m} \frac{1}{m^{5 / 3}}
$$

which is absolutely convergent.
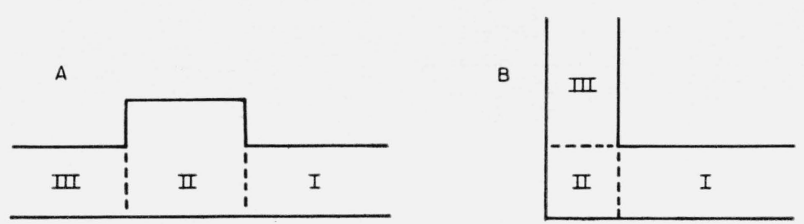

Figure 7.

\section{Concluding Remarks}

We have restricted our investigations to a firstorder approximation of the value of the reflection coefficient. Higher-order approximations are available, since we have the inverse of the matrix determining the electrostatic case.

The method of solution used in this paper may be tedious, but it is sufficiently general to be applicable to a wider range of waveguide problems. For example, it can be used in finding the field in more complex waveguide structures such as those illustrated in the following sketches.

In each of these problems there are three distinct regions, and this will give rise to two sets of reflection and transmission coefficients. (At first it might be thought that regions II and III in problem B could be combined into a single region, but elementary analysis shows that a field of this form is overdetermined and can yield only the trivial solution.)

The authors thank the referee for a large number of helpful suggestions.

\section{References}

Marcuvitz, N. (Editor) (1951), Waveguide handbook, Mass. Inst. Technol. Radiation Laboratory Series 10, (McGrawHill Book Co., Inc., New York, N.Y..).

Saxon, D. S. (1943), Notes on lectures by Julian Schwinger (MIT Radiation Laboratory Report 4304).

Magnus, W., and F. Oberhettinger (1950), On systems of linear equations in the theory of guided waves, Comm. Pure and Appl. Math. 3, 393-410.

Whittaker, E. T., and G. N. Watson (1958), A course of modern analysis (Cambridge, London).

(Paper 67D2-255) 